Bangladesh J. Bot. 48(2): 209-215, 2019 (June)

\title{
BIOCONTROL OF BASAL STEM ROT PATHOGEN GANODERMA BONINENSE BY PSEUDOMONAS AERUGINOSA
}

\author{
Pei Hui Lim, Jualang Azlan Gansau and Khim Phin Chong ${ }^{1}$ * \\ Biotechnology Programme, Faculty of Science and Natural Resources, \\ Universiti Malaysia Sabah, 88400, Jalan UMS, Kota Kinabalu, Sabah, Malaysia \\ Keywords: Biocontrol, Ganoderma boninense, Pseudomonas aeruginosa, \\ 3-Demethylubiquinone-9
}

\begin{abstract}
Ganoderma boninense is the major causal pathogens of Basal Stem Rot (BSR) of oil palm. Potential antagonists were isolated from soil samples and dual culture assays were carried out. Four types of bacteria isolates showed PIRG greater than 50\% against G. boninense and the most potential bacteria was B6 (PIRG = $75.76 \%$ ) and identified as Pseudomonas aeruginosa. The interaction between $P$. aeruginosa with $G$. boninense was observed under Scanning Electron Microscopy (SEM). Secondary metabolites of $P$. aeruginosa, were extracted using hexane, ethyl acetate and acetone. Ethyl acetate extract showed the highest inhibition towards $G$. boninense with Minimum Inhibitory Concentration (MIC) of $0.04 \mathrm{mg} / \mathrm{ml}$. Identification of compounds from the extract was further carried out using Liquid Chromatography-Mass Spectrometry (LC-MS). The most potential antimicrobial compound which may contribute to the biocontrol activity detected in the crude extract was 3-demethylubiquinone-9. P. aeruginosa and 3-demethylubiquinone-9 may potential to be further study for control of $G$. boninense.
\end{abstract}

\section{Introduction}

Basal Stem Rot (BSR) caused by Ganoderma species is the most devastating disease of oil palm in Malaysia with $G$. boninense reported as the most important species. Different methods have been used to control BSR, but to date none of the method gives good control of Ganoderma in infection states (Chong et al. 2011). Numerous endophytic bacteria can protect their host against plant pathogens through lysis or antibiotic activity. Antagonistic activity of endophytic bacteria against $G$. boninense has been studied intensively and Burkholderia cepacia, Serratia marcescens and Pseudomonas aeruginosa have been introduced as candidates for antagonistic activities (Zaiton et al. 2006). Considering biocontrol of Ganoderma boninense causal agent of basal stem rot of oil palm in Malaysia the present work was undertaken to isolate Pseudomonas aeruginosa with the highest PIRG from the soil of Crocker Range area.

\section{Materials and Methods}

Twenty soil samples were collected randomly from Crocker Range area $\left(5.4008^{\circ} \mathrm{N}, 116.1033^{\circ}\right.$ E). The isolation of microbes of interest was conducted using the dilution plate method (DPM). A serial dilution of the soil suspension from $10^{-1}$ to $10^{-8}$ was performed. An aliquot of $100 \mathrm{ul}$ of each dilution was taken and spread evenly on the surface of nutrient agar (NA) using L-shaped glass spreader. All plates were incubated at room temperature for one to two weeks. Colonies formed on the NA plates were then sub-cultured to obtain a pure culture.

G. boninense mycelial plug $(9 \mathrm{~mm})$ was taken from the edge of a seven-day-old $G$. boninense pure culture which was previously identified by Chong et al. (2011) and placed $30 \mathrm{~mm}$ from one side of the PDA plate. After two days, a loopful of the potential antagonist bacteria was streaked $30 \mathrm{~mm}$ from the G. boninense plug. G. boninense grew on PDA without the presence of antagonist

*Author for correspondence: <chongkp@ums.edu.my>. ${ }^{1}$ FGV Chair of Sustainable Oil Palm Management, Faculty of Sustainable Agriculture, UMS Sandakan Campus, Universiti Malaysia Sabah, Mile 10, Sg. Batang, 90000, Sandakan, Sabah, Malaysia 
served as control. The radial growth of the pathogen in the dual culture was measured after seven days of incubation at room temperature $\left(28 \pm 1^{\circ} \mathrm{C}\right)$ and the PIRG was calculated according to Bivi et al. (2010). Percentage Inhibition of Radial Growth (PIRG) expressed as [(R1- R2)/R1] × 100\% where, R1 is the radial growth of $G$. boninense in the control plate and R2 is the radius of the $G$. boninense colony in the direction towards the antagonist colony. Antagonists caused more than 50\% PIRG to G. boninense were labeled as effective (Ogbebor et al. 2010).

The interaction of isolate with the highest PIRG against the pathogen was observed under scanning electron microscope (Zeiss EVO® 10MA SEM). G. boninense mycelia in the middle of the agar plate were cut and then air dried in Laminar flow at room temperature for about 3 to $4 \mathrm{hrs}$. The samples were mounted onto aluminium stubs using conductive double sided adhesive carbon tabs (NISSHIN EM. CO. LTD). The stubs were then sputter coated with gold-palladium (Emitech K550x carbon coater) from different angles to ensure a complete and uniform film over sample surfaces. Coated surfaces of samples were viewed at $15 \mathrm{kV}$ voltage.

Bacteria with the highest PIRG were identified using Biolog GEN III microplate. Inoculating fluid (IF-A) was pre-warmed to room temperature before use. A colony of about $3.0 \mathrm{~mm}$ diameter was swabbed from the surface of the agar plate using a sterilized cotton bud and dipped into IF-A inoculating fluid and vortexed to obtain a uniform cell suspension before read using a turbidimeter. The turbidity must in the range of $90-98 \%$. All wells were filled with $100 \mu \mathrm{l}$ of the fluid in each well and incubated at $33^{\circ} \mathrm{C}$ ( 6 to $36 \mathrm{hrs}$ ) with the lid on. After being incubated for $24 \mathrm{hrs}$, the microplate was read using a MicroStation Reader (Biolog Inc. 2009).

Bacteria with the highest PIRG was grown in nutrient broth medium $(100 \mathrm{ml})$ for $24 \mathrm{hrs}$ and used as inoculum. For antimicrobial compounds collection, $2.0 \mathrm{ml}$ of inoculum $\left(\mathrm{OD}_{600 \mathrm{~nm}}\right.$ of 0.695$)$ was added into $1000 \mathrm{ml}$ conical flask containing $500 \mathrm{ml}$ nutrient broth for 5 days at room temperature (Sudipta and Debdulal 2010). Five-day-old broths were added with equal volume of solvent (hexane, ethyl acetate and acetone), respectively and left overnight. Cultures were then filtered using Whatman filter paper (No. 1). The solvent phase (hexane and ethyl acetate) were separated from water phase (medium) using a separatory funnel. Solvent phases were collected and dried in a rotary evaporator $\left(\right.$ Stuart $\left.^{\circledR}\right)$ at $37^{\circ} \mathrm{C}$ while the culture filtrate with acetone was collected and dried in freeze dryer (Thermo ModulyoD). The sample was pre-freezed at $-20^{\circ} \mathrm{C}$ before dried in freeze dryer. Dried crude extracts were weighed and used for bioassays. Agar dilution method $(0.20$ to $1.0 \mathrm{mg} / \mathrm{ml})$ was used to test the antimicrobial efficacy of the extracts (dissolved in $0.1 \%$ of DMSO) on G. boninense.

Minimum Inhibitory Concentration (MIC) of the bacterial extracts with the highest PIRG was determined. The concentrations tested for MIC determination were from 0.02 to $0.18 \mathrm{mg} / \mathrm{ml}$. To prepare $100 \mathrm{mg} / \mathrm{ml}$ of stock solution, $100 \mathrm{mg}$ of dried crude extract were dissolved into $1.0 \mathrm{ml}$ of dimethyl sulfoxide (DMSO) and agar preparation was carried out with different volume of stock solutions. Seven-day old G. boninense mycelial plug was placed in the center of each Petri plate. Diameter of G. boninense growth was measured for 7 days.

Identities of potential bioactive compounds from antagonist crude extracts were conducted using a Liquid Chromatography-Mass Spectrometry (LCMS). The analysis was carried out using an Acquity TM Waters Ultra Performance Liquid Chromatography (UPLC) coupled with Synapt High Definition Mass Spectrometer quadrupole-orthogonal acceleration, time-of-flight detector equipped with an electrospray ionization (ESI) source. The solvent system used was acetonitrile: water + trifluoroacetic acid $0.1 \% ; 80: 20$. The identification of the individual compounds was performed using METLIN metabolites database (https://metlin.scripps.edu/) on the basis of the mass fragments and $\mathrm{m} / \mathrm{z}$ value of each compound. 


\section{Results and Discussion}

Out of 60 bacteria isolates only 4 showed positive biocontrol with PIRG greater than 50\% against $G$. boninense. Bacteria isolate with the highest PIRG was given an identity code as B6 (PIRG $=75.76 \%)$. The dual culture assay of B6 and G. boninense is illustrated in Fig. 1 .
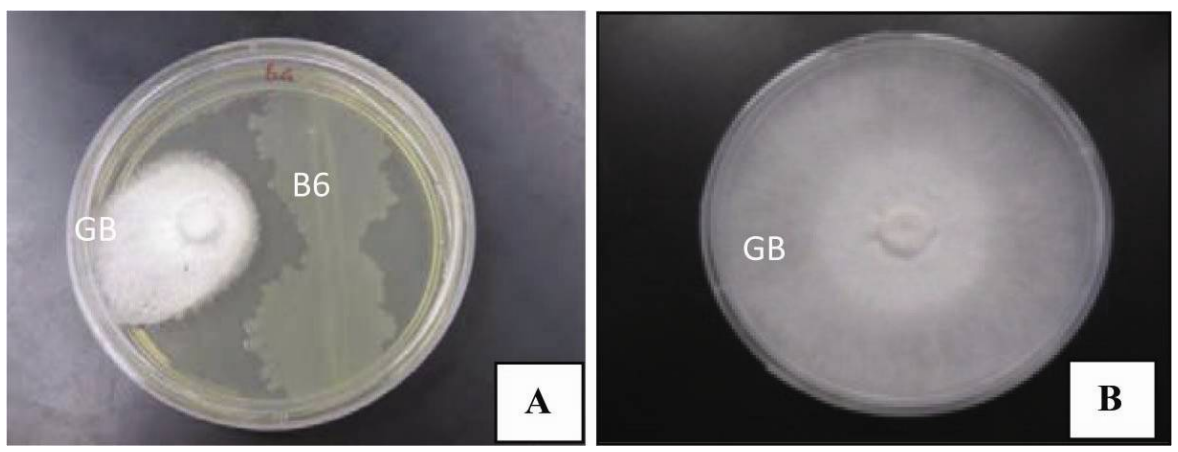

Fig. 1. Dual culture assays. A, B6, isolate with the highest PIRG (75.76\%) and B, Control plate of G. boninense. GB denotes Ganoderma boninense.

Interaction zone between B6 and $G$. boninense in dual culture assays were examined under a scanning electron microscope (SEM). A thorough examination under the SEM revealed the control G. boninense rnycelia grow healthy with dense fungal mat (Fig. 2A) while G. boninense which interacted with B6 has lower density of fungal mat and distorted hyphae could be considered as biocoltrol (Fig. 2B). B6 was identified using the Biolog identification system as Pseudomonas aeruginosa (Fig. 3).

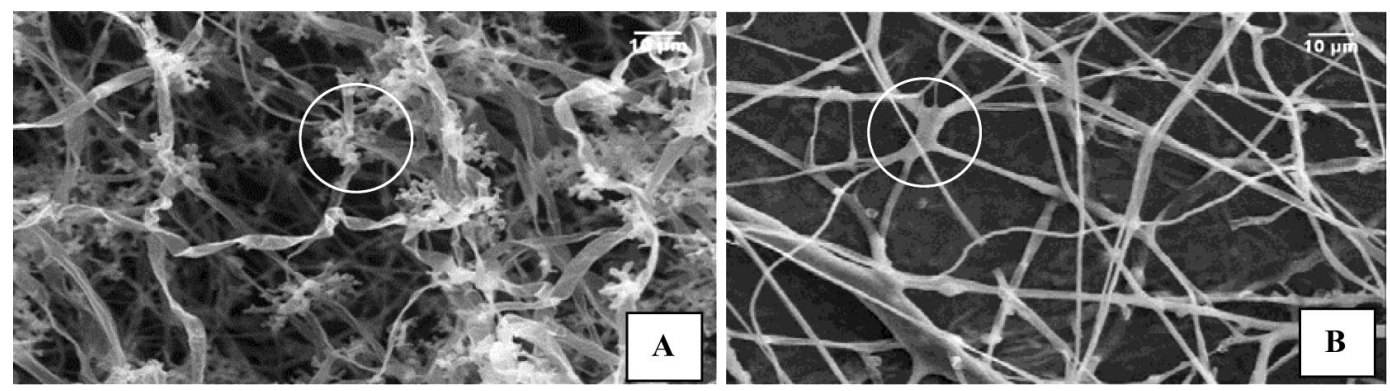

Fig. 2. Micrograph of $G$. boninense under scanning electron microscopy, A: Morphology of G. boninense hyphae without the presence of antagonist B6 (Control). B: Morphology of G. boninense hyphae interact with the presence of B6. Low fungal mat density, damage and malformation of the hyphae were observed for $G$. boninense with B6. The morphology differences between control G. boninense and $G$. boninense with antagonist were circled. Both images are magnified 2000x.

Ethyl acetate extracts of $P$. aeruginosa gave the highest inhibition (90.59\%) against $G$. boninense and differed significantly $(\mathrm{p}<0.05)$ from other solvent extracts (Fig. 4). The lowest growth inhibition was $25.33 \%$ from acetone extract. The macroscopic features of G. boninense mycelia growth in different concentrations of different extracts are shown in Fig. 5. 


ID Result
ID Comment
ID Notice

Fig. 3. Identity of B6 as $P$. aeruginosa using Biolog.

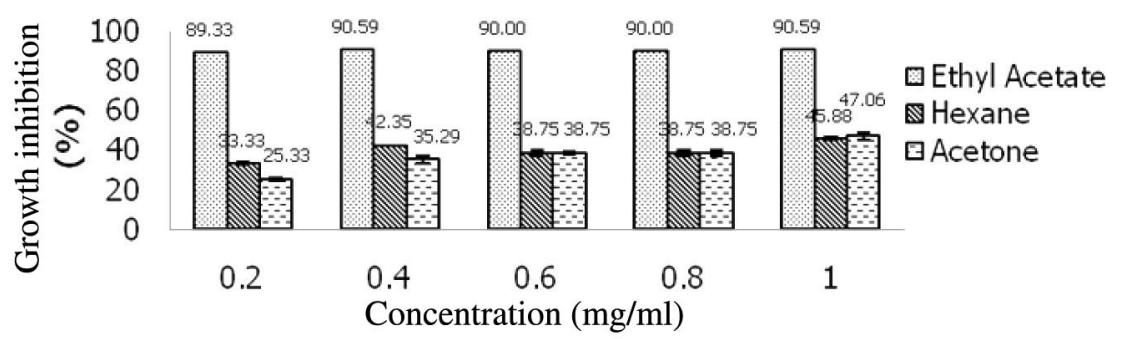

Fig. 4. Percentage inhibition of $G$. boninense growth in different concentrations of $P$. aeruginosa from various solvent extracts.
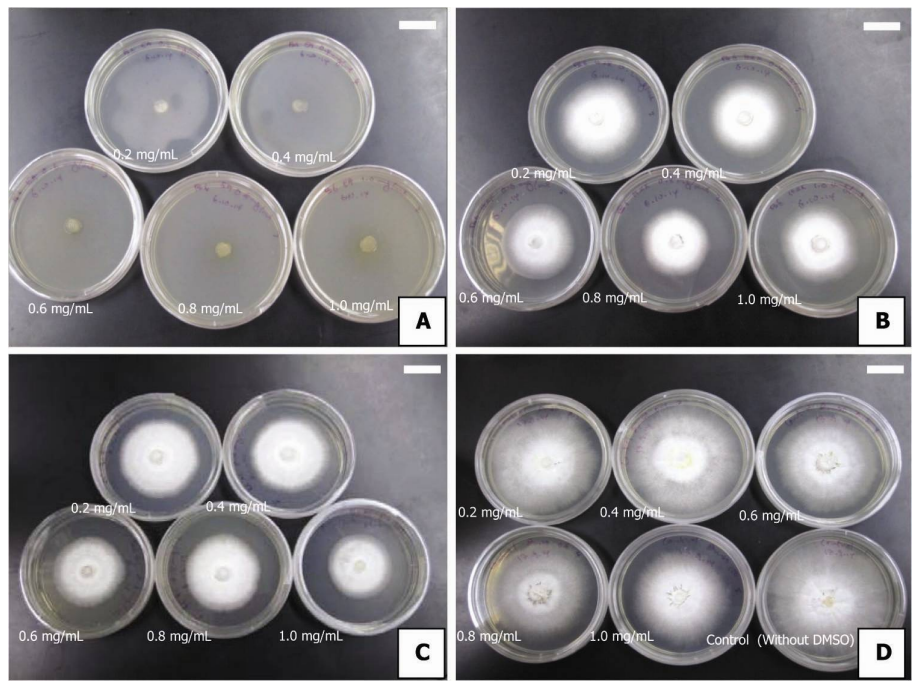

Fig. 5. Antimicrobial assays of different solvents extract of $P$. aeruginosa in 5 different concentrations. A. Ethyl acetate extract, B. Hexane extract, C. Acetone extract and D. Control (with and without DMSO). Scale bar: $2 \mathrm{~cm}$. 
The $G$. boninense growth treated with different concentrations of $P$. aeruginosa ethyl acetate crude extracts from day one to day seven is shown in Table 1. There was no visible growth of $G$. boninense observed at concentration of 0.04 to $0.20 \mathrm{mg} / \mathrm{ml}$. Thus, MIC value for P. aeruginosa extract was estimated at $0.04 \mathrm{mg} / \mathrm{l}$.

Table 1. The growth diameter $(\mathrm{cm})$ of $G$. boninense in different concentrations of $P$. aeruginosa ethyl acetate extracts.

\begin{tabular}{|c|c|c|c|c|c|c|c|}
\hline $\begin{array}{l}\text { Conc. } \\
(\mathrm{mg} / \mathrm{ml})\end{array}$ & Day 1 & Day 2 & Day 3 & Day 4 & Day 5 & Day 6 & Day 7 \\
\hline 0.02 & $0.80 \pm 0.00$ & $0.80 \pm 0.00$ & $0.80 \pm 0.00$ & $0.80 \pm 0.00$ & $0.80 \pm 0.00$ & $1.63 \pm 0.00$ & $2.23 \pm 0.00$ \\
\hline 0.04 & $0.80 \pm 0.00$ & $0.80 \pm 0.00$ & $0.80 \pm 0.00$ & $0.80 \pm 0.00$ & $0.80 \pm 0.00$ & $0.80 \pm 0.00$ & $0.80 \pm 0.00$ \\
\hline 0.06 & $0.80 \pm 0.00$ & $0.80 \pm 0.00$ & $0.80 \pm 0.00$ & $0.80 \pm 0.00$ & $0.80 \pm 0.00$ & $0.80 \pm 0.00$ & $0.80 \pm 0.00$ \\
\hline 0.08 & $0.80 \pm 0.00$ & $0.80 \pm 0.00$ & $0.80 \pm 0.00$ & $0.80 \pm 0.00$ & $0.80 \pm 0.00$ & $0.80 \pm 0.00$ & $0.80 \pm 0.00$ \\
\hline 0.10 & $0.80 \pm 0.00$ & $0.80 \pm 0.00$ & $0.80 \pm 0.00$ & $0.80 \pm 0.00$ & $0.80 \pm 0.00$ & $0.80 \pm 0.00$ & $0.80 \pm 0.00$ \\
\hline 0.12 & $0.80 \pm 0.00$ & $0.80 \pm 0.00$ & $0.80 \pm 0.00$ & $0.80 \pm 0.00$ & $0.80 \pm 0.00$ & $0.80 \pm 0.00$ & $0.80 \pm 0.00$ \\
\hline 0.14 & $0.80 \pm 0.00$ & $0.80 \pm 0.00$ & $0.80 \pm 0.00$ & $0.80 \pm 0.00$ & $0.80 \pm 0.00$ & $0.80 \pm 0.00$ & $0.80 \pm 0.00$ \\
\hline 0.16 & $0.80 \pm 0.00$ & $0.80 \pm 0.00$ & $0.80 \pm 0.00$ & $0.80 \pm 0.00$ & $0.80 \pm 0.00$ & $0.80 \pm 0.00$ & $0.80 \pm 0.00$ \\
\hline 0.18 & $0.80 \pm 0.00$ & $0.80 \pm 0.00$ & $0.80 \pm 0.00$ & $0.80 \pm 0.00$ & $0.80 \pm 0.00$ & $0.80 \pm 0.00$ & $0.80 \pm 0.00$ \\
\hline 0.20 & $0.80 \pm 0.00$ & $0.80 \pm 0.00$ & $0.80 \pm 0.00$ & $0.80 \pm 0.00$ & $0.80 \pm 0.00$ & $0.80 \pm 0.00$ & $0.80 \pm 0.00$ \\
\hline
\end{tabular}

Based on the LC-MS analysis (Fig. 6), compounds identified were further selected based on published results on their antimicrobial activity. The potential antimicrobial compound detected in $P$. aeruginosa ethyl acetate crude extract was 3-demethylubiquinone-9 with $\mathrm{m} / \mathrm{z} 763.60[\mathrm{M}+\mathrm{H}-$ $\mathrm{H} 2 \mathrm{O}]^{+}$.
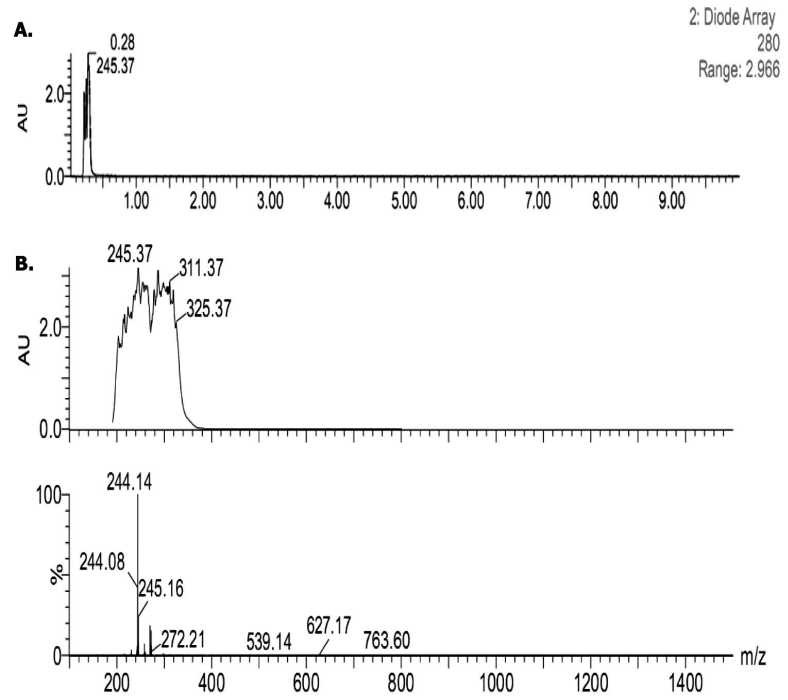

Fig. 6 A. Chromatogram of $P$. aeruginosa ethyl acetatecrude extract from LC-MS analysis. B, The extracted UV and mass spectra at $t_{R} 0.28 \mathrm{~min}$. Potential antimicrobial compound detected in the mass spectra as 3-demethylubiquinone-9 [M+H-H2O $]^{+} \mathrm{m} / \mathrm{z} 763.60$. 
Borneo is the second largest tropical island in the world, harboring precious biodiversity resources. Crocker Range Park, an area under the Bornean biodiversity and ecosystem Conservation (BBEC) program where the soil samples were collected may increase the possibility in isolation potential antagonists against $G$. boninense. Forest soil contains thousands of species of microorganisms and has higher microbial population compared to disturbed plantation soil (Staddon et al. 1996). Forest vegetation influences the micro-environmental conditions and energy supply through their root system, and above ground and below ground biological processes also affect soil microorganisms (Merila et al. 2002, Zhang et al. 2005). The relatively dense growth of plants and higher accumulation of litter on the forest floor and distribution of fine roots in undisturbed forest favor the growth of microorganisms (Dkhar et al. 2012).

The dual culture assays revealed that the growth of $G$. boninense mycelium was stunted with the presence of $P$. aeruginosa. The secondary metabolites of $P$. aeruginosa may penetrate the pathogen cells and inhibit the activity by chemical toxicity. However, despite the inhibition of fungal growth, neither lysed hyphal tips nor collapsed hyphae due to the presence of $P$. aeruginosa were observed upon SEM examination. Only damaged hyphae were observed within the mycelium and the growth of antler-like hyphae were inhibited. Pseudomonades have been reported to produce a wide spectra of compounds with antimicrobial activity against phytopathogenic fungi and bacteria (Cazorla et al. 2006). Pseodomonades strains often produce more than one antimicrobial compound such as different phenazines, phenazine-1-carboxylic acid and 2,4-diacethylploroglucinol, pyoluteorin and pyrrolnitrin (Paul and Sarma 2006), monoacetylphloroglucinol and 2,4-diacethylploroglucinol (Guihen et al. 2004). However only one notable compound 3-demathylubiquinone-9 which may contribute to the antifungal activity against $G$. boninense was detected in this work. The ethyl acetate extract had effectively inhibited the pathogen. This could be related to the presence of 3-demathylubiquinone-9 which is soluble in ethyl acetate and moderately polar to polar in nature.

3-demethylubiquinone-9 is a polyprenylhydroquinone which has a structure of ubiquinol-9 with hydroxy replacing methoxy at C-5. New quinone and hydroquinone antibiotics produced by Pseudomonas spp. were reported to be active against Gram positive bacteria, some fungi and yeasts (Kumagai et al. 1992). The antifungal activity of these antibiotics was characterized by the specific inhibitory action against fungal cell wall synthesis. These antibiotics were active againsts several fungi species such as Trichophyton mentagrophytes, Aspergillus fumigatus and Cryptococcus neoformans. Giving the unique nature of the fungal cell wall, any disruption in cell wall integrity should affect the growth (Selitrennikoff and Nakata 2003). It is therefore suggested that the similar mechanism may affect the growth of G. boninense by the isolated Pseudoimones spp. in the present study.

B6 isolate which gave the highest PIRG (75.76\%) against $G$. boninense was identified as $P$. aeruginosa using Biolog. Ethyl acetate crude extract of $P$. aeruginosa gave the best inhibitory effect against G. boninense with MIC of $0.04 \mathrm{mg} / \mathrm{ml}$. The compound which may contribute to this biocontrol effect was identified as 3-demethylubiquinone-9 using LC-MS.

\section{Acknowledgements}

The authors like to express their deepest gratitude to the authorities of the Sabah State Government for the scholarship offered to Pei Hui Lim under Sabah State Government Scholarship Scheme. This research was funded by ERGS (ERGS0017-STG-1/2012) from Ministry of Higher Education, Malaysia and the PhD Incentive Grant (PhD0001-SG-1/2012) of Universiti Malaysia Sabah. 


\section{References}

Biolog Inc. 2009. MicroStation ${ }^{\mathrm{TM}}$ System/MicroLog User's Guide Version 5.1.1. USA. Section 4, Pg. 15.

Bivi MR, Farhana MSN, Khairulmazmi A and Idris AS 2010. Control of Ganoderma boninense: A causal agent of basal stem rot disease in oil palm with endophyte bacteria in vitro. Int. J. Agric. Biol. 12: 833839.

Cazorla FM, Duckett SB, Bergstroem ET, Noreen S, Odijk R, Lugtenberg BJ, Thomas-Oates JE and Bloemberg GV 2006. Biocontrol of avocado dematophora root rot by antagonistic Pseudomonas fluorescens PCL1606 correlates with the production of 2-hexyl 5-propyl Resorcinol. Mol Plant Microbe Interact. 19: 418-428.

Chong KP, Lum MS, Foong CP, Wong CMVL, Atong M and Rossall S 2011. First identification of Ganoderma boninense isolated from Sabah based on pcr and sequence homology. Afr. J. Biotechnol. 10(66): 14718-14723.

DkharM, Dkhar MS and Tynsong H 2012. Microbial activities and nutrient dynamics in sacred forest of Meghalaya. India J. Inn. Dev.1(3): 175-185.

Guihen E, Glennon JD, Cullinane M and O' Gara F 2004. Rapid analysis of antimicrobial metabolites monoacetylphloroglucinol and 2,4-diacetylphoro-glucinol using capillary zone electrophoresis. Electrophoresis 25: 1536-1542.

Kumagai K, Kato M, Nabeshima S and Tsuyumu S 1992. T 1801 A, B, C, and D, new quinone and hydroquinone antibiotics produced by a strain of Pseudomonas. Biosci, Biotechnol. and Biochem. 56(9): 1439-1442.

Merila P, Strommer R and Fritze H 2002. Soil microbial activity and community structure along a primary succession transect on the land-uplift coast in western Finland. Soil Biol. and Biochem. 14: 1647-1654.

Ogbebor NO,Adekunle AT, Eghafona NO and Ogboghodo AI 2010. Ganoderma psuedoferreum: Biological control possibilities with microorganisms isolated from soils of rubber plantationsin Nigeria. Afr. J. Gen. Agric. 6(4): 303.

Paul D and Sarma YR 2006. Plant growth promoting rhizhobacteria (PGPR) mediated root proliferation in black pepper (Piper nigrum L.) as evidenced through GS root software. Arch. Phyto. Plant Protect. 39: 311-314.

Selitrennikoff CP and Nakata M 2003. New cell wall targets for antifungal drugs. Curr. Opin. Investig. Drugs 4(2): 200- 205.

Sudipta R and Debdulal B 2010. Isolation of antimicrobial compound by endophytic bacteria from Vincarosea. Int. J. Curr. Res. 5: 47-51.

Staddon WJ, Duchesne LC and Trevors JT 1996. Conservation of forest soil microbial diversity: The impact of fire and research needs environmental review 4: 267-75.

Zaiton S, Sariah M and Zainal AMA 2006. Isolation and characterization of microbial endophytes from oil palm roots: Implication as biological control agents against Ganoderma. Planter. 82(966): 587-597.

Zhang W, Wei H, Gao H and Hu Y 2005. Advances of studies on soil microbial diversity and environmental impact factors. Chi. J. Eco. 24: 48-52. (In Chinese). 